\title{
Perioperative Evaluation of Respiratory Muscle Strength after Scoliosis Correction in Patients with Duchenne Muscular Dystrophy
}

\author{
Wataru Saito ${ }^{1}$, Kosuke Mizuno ${ }^{2}$, Gen Inoue ${ }^{1}$, Takayuki Imura ${ }^{1}$, Toshiyuki Nakazawa ${ }^{1}$, \\ Masayuki Miyagi ${ }^{1}$, Eiki Shirasawa ${ }^{1}$, Kentaro Uchida $^{1}$, Masashi Takaso ${ }^{1}$ \\ ${ }^{I}$ Department of Orthopaedic Surgery, Kitasato University School of Medicine, Sagamihara, Japan \\ ${ }^{2}$ Department of Rehabilitation, Kitasato University Hospital, Sagamihara, Japan
}

\section{Study Design: Retrospective cohort study.}

Purpose: To investigate the effect of spinal correction on respiratory muscle strength in patients with Duchenne muscular dystrophy (DMD).

Overview of Literature: Several studies have reported that scoliosis correction in patients with DMD does not improve pulmonary function. In these studies, pulmonary function was evaluated using the traditional spirometric values of percent vital capacity (\%VC) and percent forced vital capacity (\%FVC). However, traditional spirometry may not be suitable for patients with DMD because the results can be influenced by patient fatigue or level of understanding. Therefore, we evaluated respiratory function focusing on respiratory muscle strength using maximal inspiratory pressure (MIP), maximal expiratory pressure (MEP), and sniff nasal inspiratory pressure (SNIP), in addition to \%VC and \%FVC.

Methods: We retrospectively reviewed 16 patients with DMD who underwent spinal correction surgery between 2006 and 2011 at Kitasato University Hospital. All patients were males, and the mean age was 13.5 years. Respiratory muscle strength was evaluated using MIP, MEP, and SNIP. Measurements were obtained preoperatively and at 1 and 6 months postoperatively, and \%VC and \%FVC were obtained preoperatively and within 6 months postoperatively.

Results: The mean preoperative and postoperative \%VC values were $54.0 \%$ and $51.7 \%$, whereas the mean $\% \mathrm{FVC}$ values were $53.9 \%$ and $53.2 \%$, respectively. The mean MIP, MEP, and SNIP values obtained preoperatively and at 1 and 6 months postoperatively were as follows: MIP, 40.5, 42.7 and $47.2 \mathrm{~cm} \mathrm{H}_{2} \mathrm{O}$; MEP, 26.0, 28.0, and $29.0 \mathrm{~cm} \mathrm{H}_{2} \mathrm{O}$; and SNIP, 33.4, 33.0, and $33.0 \mathrm{~cm} \mathrm{H}_{2} \mathrm{O}$; respectively. The mean MIP and MEP values significantly improved postoperatively. There were no significant differences in SNIP, \%VC, or \%FVC preand postoperatively.

Conclusions: By focusing on respiratory muscle strength, our results suggest that scoliosis correction in patients with DMD might have a favorable effect on respiratory function.

Keywords: Respiratory muscle; Duchenne muscular dystrophy; Pulmonary function; Scoliosis correction

Received Feb 15, 2017; Revised Feb 22, 2017; Accepted Feb 23, 2017

Corresponding author: Wataru Saito

Department of Orthopedics, Kitasato University School of Medicine, 1-15-1 Kitasato, Minami-ku, Sagamihara 252-0373, Japan

Tel: +81-42-778-8707, Fax: +81-42-778-585, E-mail: boatwataru0712@gmail.com 


\section{Introduction}

Patients with Duchenne muscular dystrophy (DMD) commonly develop scoliosis after loss of ambulation [1]. Most cases are progressive [2,3], and operative treatment such as scoliosis correction and fusion is considered for all except mild cases [4-8]. Many case-control studies and case series have reported that scoliosis correction has beneficial effects on sitting balance $[9,10]$, quality of life (QOL) $[5,8,11,12]$, cosmesis, and self-image $[12,13]$. However, its effect on pulmonary function remains controversial.

Previous studies have reported that scoliosis correction improves respiratory function and prevents further respiratory decline $[2,14]$. However, several other studies have demonstrated no obvious benefit in terms of respiratory function $[5,9,10,15-18]$. In these studies, respiratory function was evaluated using percent vital capacity (\%VC) and percent forced vital capacity (\%FVC). These tests are strongly influenced by a patient's level of understanding, motivation, and fatigue and may be difficult for patients with DMD to perform. Alternatively, maximum inspiratory pressure (MIP), maximal expiratory pressure (MEP), and sniff nasal inspiratory pressure (SNIP) have been reported to be useful in evaluating respiratory muscle strength in patients with DMD [19-22]. However, no previous reports have evaluated respiratory function using these methods in patients with DMD following scoliosis correction.

In this study, we evaluated perioperative respiratory muscle strength using MIP, MEP, and SNIP, in addition to conventional evaluation methods, to clarify whether scoliosis correction in patients with DMD can improve respiratory function.

\section{Materials and Methods}

Approval for this study and for the publication of this manuscript was obtained from the Institutional Review Board of Kitasato University School of Medicine. We retrospectively reviewed the cases of 16 patients with DMD who were surgically treated at Kitasato University Hospital from 2006 to 2011. All patients underwent posterior spinal correction with pedicle screws and sublaminar wiring. The spinal fusion level was between T4 and L5 in all patients. The mean age at the time of surgery was 13.5 years (range, 11-16 years), and the mean body mass index was $20.2 \mathrm{~kg} / \mathrm{m}^{2}$ (range, $11.3-24.9 \mathrm{~kg} / \mathrm{m}^{2}$ ). Patient demographic
Table 1. Patients' demographic and radiographic data

\begin{tabular}{lc} 
Characteristic & Mean \pm Standard deviation (range) \\
Patients number & 16 \\
\hline Age $(\mathrm{yr})$ & $13.5 \pm 1.5(11-16)$ \\
\hline Sex & Male 16 \\
\hline Height $(\mathrm{cm})$ & $149.4 \pm 7.6(130-165)$ \\
Weight $(\mathrm{kg})$ & $46.1 \pm 14.9(20-74)$ \\
\hline Body mass index $\left(\mathrm{kg} / \mathrm{m}^{2}\right)$ & $20.2 \pm 5.1(11.3-24.9)$ \\
Pre Cobb $\left(^{\circ}\right)$ & $73.4 \pm 23.8(46-119)$ \\
\hline Post Cobb $\left(^{\circ}\right)$ & $26.1 \pm 12.7(1-45)$ \\
Correction rate $(\%)$ & $64.4 \pm 15.0(37.5-98.1)$ \\
\hline
\end{tabular}

data are summarized in Table 1. The spinal curve was measured using the Cobb angle. Respiratory function was measured using \% $\mathrm{VC}$ and $\% \mathrm{FVC}$, and respiratory muscle strength was measured using MIP, MEP, and SNIP. These parameters were measured by a physical therapist using a RPM01 (CareFusion Co. Ltd., San Diego, CA, USA), with the patients in the sitting position. After ensuring that the bilateral nasal cavities were not obstructed, a mensurative probe was unilaterally inserted into the nasal cavity to measure SNIP. The patients were then instructed to close their mouth and draw in a breath strongly and quickly, starting from the functional residual capacity. The signal from the probe was measured using pressure instrumentation, and the pressure curve was monitored using a personal computer. These series of maneuvers were performed five times in 30-second intervals. To measure MIP, the patients were instructed to strongly draw in a breath at maximal end-tidal volume with the bilateral nasal cavities closed, and the physical therapist measured the intraoral pressure using a mensurative mouthpiece. The MEP values were measured using the same method as MIP, but the patients were instructed to strongly expire from the maximal end of inspiration. These series of maneuvers were performed at least three times in 30-second intervals. Respiratory muscle strength was measured in this manner preoperatively and at 1 and 6 months postoperatively.

The \%VC and \%FVC values measured by clinical laboratory technicians preoperatively and within 6 months postoperatively were obtained from the clinical records.

Differences between preoperative and postoperative data were analyzed using paired $t$-test with the SPSS Statistics ver. 19 software package (IBM, Tokyo, Japan). Data are shown as mean \pm standard deviation. A $p$-value of 
$<0.05$ was considered statistically significant.

\section{Results}

The mean preoperative Cobb angle was $73.4^{\circ} \pm 21.6^{\circ}$ (range, $46^{\circ}-119^{\circ}$ ), which was corrected to $24.9^{\circ} \pm 11.2^{\circ}$ (range, $1^{\circ}-45^{\circ}$ ) postoperatively. The mean correction rate was $67.1 \% \pm 15.7 \%$ (range, $50.0 \%-98.1 \%$ ). Radiographic data are summarized in Table 1 . The mean MIP values obtained preoperatively and at 1 and 6 months postoperatively were $40.5 \pm 13.8,42.7 \pm 16.5$, and $47.2 \pm 14.5 \mathrm{~cm}$ $\mathrm{H}_{2} \mathrm{O}$, respectively, showing significant improvement at 6 months postoperatively (Fig. 1). The mean MEP values obtained preoperatively and at 1 and 6 months postoperatively were $26.0 \pm 8.5,28.3 \pm 11.5$, and $29.3 \pm 11.8 \mathrm{~cm} \mathrm{H}_{2} \mathrm{O}$, respectively, showing slight but not significant improve-

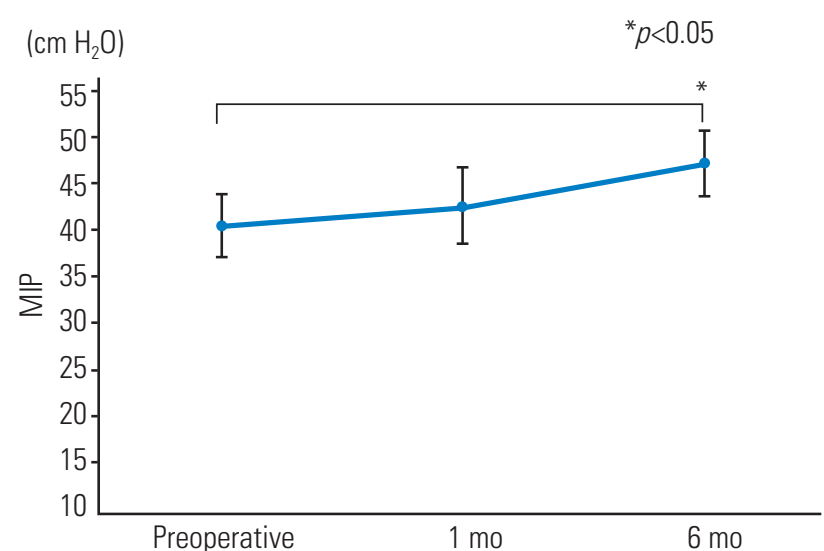

Fig. 1. Mean maximum inspiratory pressure (in $\mathrm{cm} \mathrm{H}_{2} \mathrm{O}$ ) preoperatively and at 1 and 6 months postoperatively. The error bars in the graph indicate standard error. MIP, maximal inspiratory pressure.

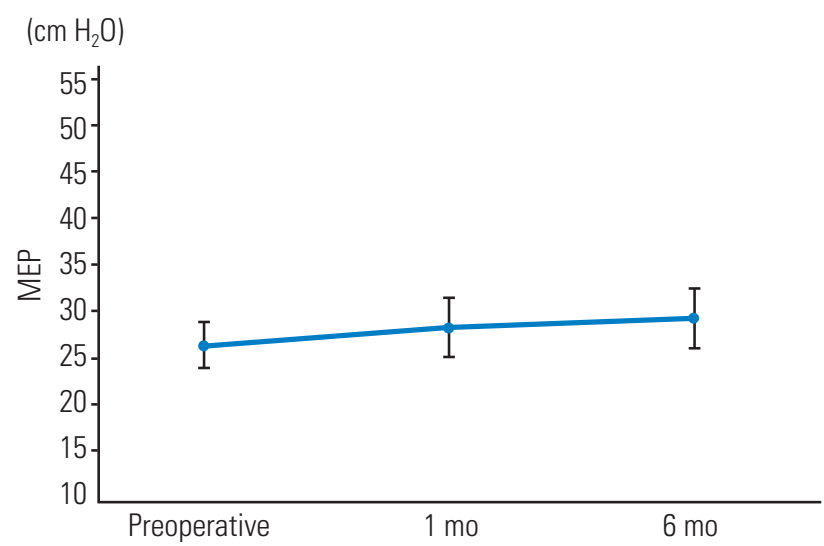

Fig. 2. Mean maximum expiratory pressure (in $\mathrm{cm}_{2} \mathrm{O}$ ) preoperatively and at 1 and 6 months postoperatively. The error bars in the graph indicate standard error. MEP, maximal expiratory pressure. ment at 1 and 6 months postoperatively (Fig. 2). The mean SNIP values obtained preoperatively and at 1 and 6 months postoperatively were $33.4 \pm 13.1,33.2 \pm 12.7$, and $32.7 \pm 12.7 \mathrm{~cm} \mathrm{H}_{2} \mathrm{O}$, respectively, showing no improvement postoperatively (Fig. 3). The mean pre- and postoperative $\%$ VC values were $49.2 \% \pm 16.2 \%$ and $50.1 \% \pm 18.6 \%$, respectively, and the mean $\% \mathrm{FVC}$ values were $53.9 \% \pm 12.8 \%$ and $53.3 \% \pm 12.3 \%$, respectively; $\% \mathrm{VC}$ tended to increase and \%FVC showed no improvement (Figs. 4, 5).

\section{Discussion}

We evaluated the effect of scoliosis correction on patients with DMD using respiratory muscle strength tests. Although there was no specific difference in the preoperative

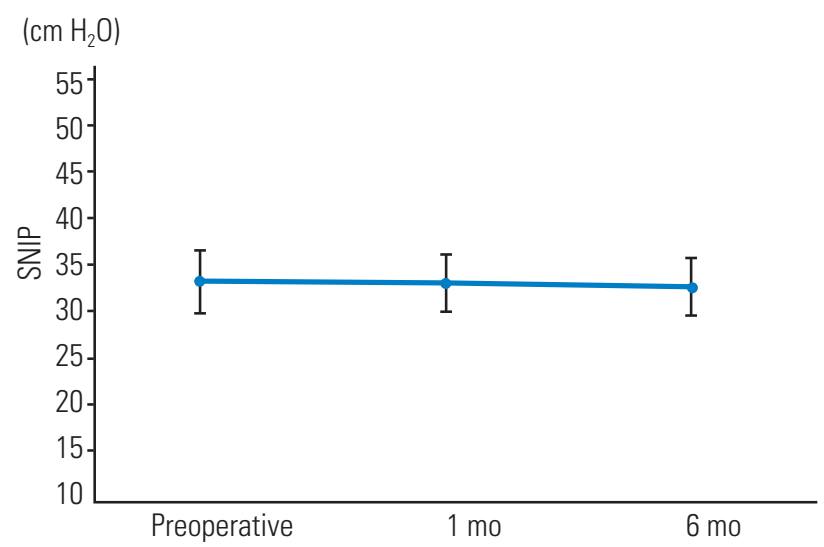

Fig. 3. Mean sniff nasal inspiratory pressure (in $\mathrm{cm}_{2} \mathrm{O}$ ) preoperatively and at 1 and 6 months postoperatively. The error bars in the graph indicate standard error. SNIP, sniff nasal inspiratory pressure.

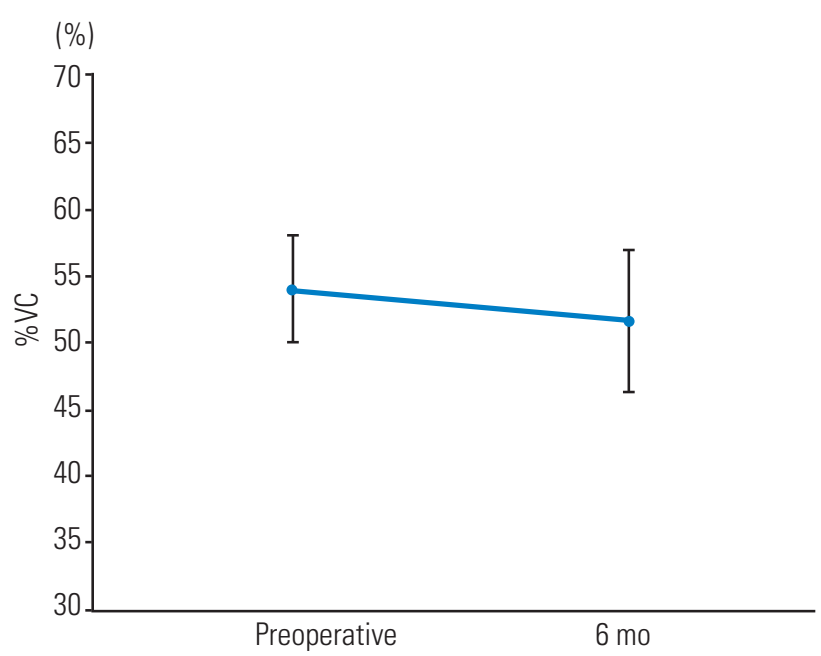

Fig. 4. Mean \%VC values preoperatively and at 6 months postoperatively. The error bars in the graph indicate standard error. \%VC, percent vital capacity. 


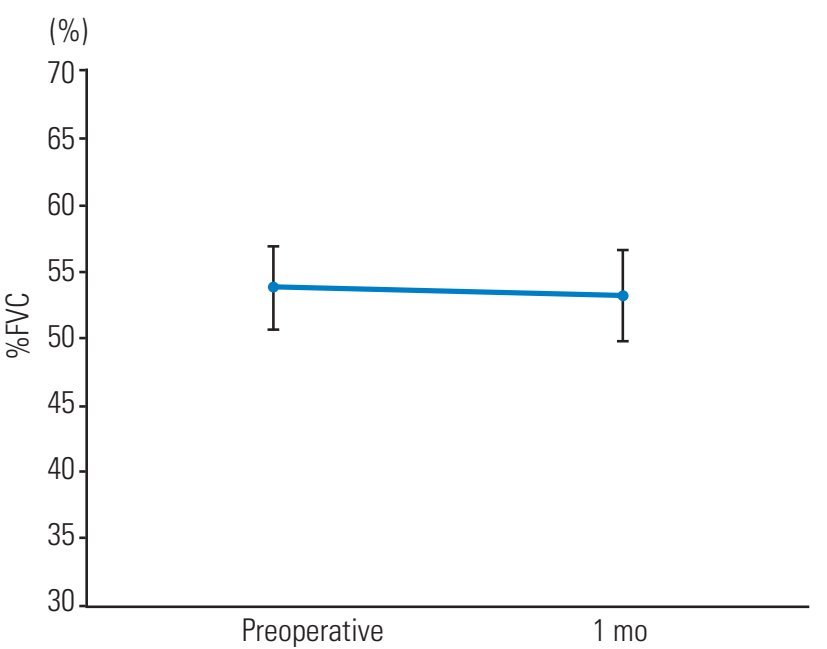

Fig. 5. Mean \%FVC values preoperatively and at 6 months postoperatively. The error bars in the graph indicate standard error. \%FVC, percent forced vital capacity.

and postoperative $\% \mathrm{VC}$ and $\% \mathrm{FVC}$ values, we observed small improvements in MEP at 1 and 6 months postoperatively and a significant improvement in MIP 6 months postoperatively. Our results suggest that scoliosis correction improves postoperative respiratory muscle strength in patients with DMD.

Currently, spinal correction and fusion surgery is the mainstay of scoliosis treatment in patients with DMD. The advantages of surgery include increased comfort and sitting tolerance $[10,12,23]$, increased upper extremity function [23,24], easier nursing care by parents [12], increased QOL $[8,11,12]$, cosmetic improvement, and higher selfimage $[12,13]$.

However, the effect of scoliosis correction on pulmonary function remains controversial. Progressive deterioration of respiratory function occurs during the natural course of DMD because of weakening of the respiratory muscles. In addition, deterioration of respiratory function appears to be related to progression of scoliosis [4, 25-28]. After the onset of spinal deformity, scoliosis can rapidly progress and compromise respiratory function [28]. Several groups have thus investigated the effect of scoliosis correction on respiratory function. Several studies have reported the stabilization of VC, FVC, and peak expiratory flow rate or slowing of the rate of \%FVC decline in surgically treated patients $[2,14]$. In contrast, many studies have reported that scoliosis correction does not improve respiratory function in patients with DMD $[5,6,9,10,15-18]$. In these studies, respiratory function was evaluated using traditional spirometric values such as VC, forced expiratory volume in 1 second, and peak expiratory flow. These values evaluate respiratory function as a whole, including thoracic compliance and respiratory muscle strength. Furthermore, the results of these tests are easily affected by patient fatigue, motivation, and level of understanding. Thus, traditional spirometry might not be suitable for evaluating the effect of scoliosis correction on respiratory function in patients with neuromuscular diseases such as DMD.

Measurements of respiratory muscle strength, such as MIP, MEP, and SNIP, have been used to evaluate respiratory function in diseases such as chronic obstructive pulmonary disease and neuromuscular disease [19]. Nava et al. [20] used MIP, SNIP, and combined maneuvers (adding MEP after MIP) to differentiate between the respiratory muscle strength of the rib cage, abdomen, and respiratory muscles. Stefanutti et al. [22] demonstrated the feasibility and usefulness of SNIP in a large group of children and adults with various neuromuscular and skeletal disorders. Furthermore, Nicot et al. [21] reported that in 39\% of young patients with neuromuscular disease, FVC could not be measured and that SNIP was a more valuable tool for the assessment of respiratory muscle strength in this patient population. These studies suggest that MIP, MEP, and SNIP are suitable tests of respiratory function in children with neuromuscular diseases. In our study, almost all patients were able to perform respiratory muscle strength tests (MIP, MEP, and SNIP) without difficulty (in three patients, MEP could not be measured because of an air leak; the size of the mouthpiece did not fit the patient's mouth).

In the present study, respiratory muscle strength was measured using MIP, MEP, and SNIP and respiratory function was measured using conventional perioperative tests (\%VC and \%FVC) in patients with DMD who underwent scoliosis correction. Our data are consistent with the findings of previous studies. We observed no significant postoperative improvement in either \% $\mathrm{VC}$ or $\% \mathrm{FVC}$, which supports the view that scoliosis correction does not play a role in the improvement of respiratory function. On the other hand, both MIP and MEP improved postoperatively. Although the exact mechanism has not been fully elucidated, we believe that the deterioration of sitting balance, development of thoracic cavity deformity, and decrement of thoracic cavity compliance due to spinal deformity may negatively influence rib movement and weaken respiratory muscles, including the internal/external intercostal muscles, abdominal rectus muscle, abdominal 
internal/external oblique muscles, and diaphragm. We speculate that spinal correction improves sitting balance and thoracic deformity, thereby positively influencing respiratory muscle movement. Respiratory muscle strength measurements are capable of detecting these improvements in respiratory muscle movement more sensitively and specifically than conventional pulmonary function tests in patients with neuromuscular scoliosis. Because of these reasons, the respiratory muscle strength values and conventional pulmonary function test results changed differently after spinal correction.

Although MIP and SNIP are both influenced by inspiratory muscle strength, MIP significantly improved postoperatively but SNIP did not. Previous reports have suggested that the agreement between these two tests is relatively poor [20]. Stefanutti et al. [22] pointed out that because of differences in the type of effort and the pattern of muscle activation required by the two maneuvers, the values obtained from MIP and SNIP maneuvers probably reflect different aspects of inspiratory muscle function. It has also been reported that MIP and MEP entirely reflect the strength of the inspiratory and expiratory muscles, respectively, whereas SNIP mainly reflects the contractile strength of the diaphragm [20]. Therefore, the results of this study support the speculation that in the short term, spinal correction can influence inspiratory/expiratory muscle strength rather than diaphragm strength.

It is reported that preoperative respiratory muscle training contributes to pulmonary function improvement [23]. Based on our results, respiratory muscle training particularly focusing on inspiratory and expiratory muscles could produce a positive effect on pulmonary function and possibly reduce perioperative respiratory complications and effectively maintain pulmonary function.

This was a short-term study with a relatively small number of patients. Hence, it remains uncertain whether our findings will be applicable to a larger population. Adequately designed studies with a larger number of patients may help answer this question. Nonetheless, the results of this study may be useful in designing perioperative respiratory rehabilitation programs that slow down the natural deterioration of respiratory function in patients with DMD.

\section{Conclusions}

We evaluated postoperative improvements in respiratory muscle strength using MIP, MEP, and SNIP in patients with DMD who underwent spinal correction surgery. The mean MIP and MEP values improved postoperatively. Therefore, our results suggest that respiratory muscle strength improves after scoliosis correction in patients with DMD.

\section{Conflict of Interest}

No potential conflict of interest relevant to this article was reported.

\section{Acknowledgments}

This work was supported in part by an Intramural Research Grant (26-6) for Neurological and Psychiatric Disorders from the National Center of Neurology and Psychiatry.

\section{References}

1. Gardner-Medwin D. Clinical features and classification of the muscular dystrophies. Br Med Bull 1980;36:109-15.

2. Galasko CS, Williamson JB, Delaney CM. Lung function in Duchenne muscular dystrophy. Eur Spine J 1995;4:263-7.

3. Miller F, Moseley CF, Koreska J, Levison H. Pulmonary function and scoliosis in Duchenne dystrophy. J Pediatr Orthop 1988;8:133-7.

4. Galasko CS, Delaney C, Morris P. Spinal stabilisation in Duchenne muscular dystrophy. J Bone Joint Surg Br 1992;74:210-4.

5. Granata C, Merlini L, Cervellati S, et al. Long-term results of spine surgery in Duchenne muscular dystrophy. Neuromuscul Disord 1996;6:61-8.

6. Hahn F, Hauser D, Espinosa N, Blumenthal S, Min K. Scoliosis correction with pedicle screws in Duchenne muscular dystrophy. Eur Spine J 2008;17:255-61.

7. Heller KD, Wirtz DC, Siebert CH, Forst R. Spinal stabilization in Duchenne muscular dystrophy: principles of treatment and record of 31 operative treated cases. J Pediatr Orthop B 2001;10:18-24.

8. Marchesi D, Arlet V, Stricker U, Aebi M. Modification of the original Luque technique in the treatment of Duchenne's neuromuscular scoliosis. J Pediatr Orthop 1997;17:743-9. 
9. Miller RG, Chalmers AC, Dao H, Filler-Katz A, Holman D, Bost F. The effect of spine fusion on respiratory function in Duchenne muscular dystrophy. Neurology 1991;41:38-40.

10. Shapiro F, Sethna N, Colan S, Wohl ME, Specht L. Spinal fusion in Duchenne muscular dystrophy: a multidisciplinary approach. Muscle Nerve 1992;15:604-14.

11. Bentley G, Haddad F, Bull TM, Seingry D. The treatment of scoliosis in muscular dystrophy using modified Luque and Harrington-Luque instrumentation. J Bone Joint Surg Br 2001;83:22-8.

12. Bridwell KH, Baldus C, Iffrig TM, Lenke LG, Blanke K. Process measures and patient/parent evaluation of surgical management of spinal deformities in patients with progressive flaccid neuromuscular scoliosis (Duchenne's muscular dystrophy and spinal muscular atrophy). Spine (Phila Pa 1976) 1999;24:1300-9.

13. Chong HS, Moon ES, Kim HS, et al. Comparison between operated muscular dystrophy and spinal muscular atrophy patients in terms of radiological, pulmonary and functional outcomes. Asian Spine J 2010;4:82-8.

14. Velasco MV, Colin AA, Zurakowski D, Darras BT, Shapiro F. Posterior spinal fusion for scoliosis in duchenne muscular dystrophy diminishes the rate of respiratory decline. Spine (Phila $\mathrm{Pa} 1976$ ) 2007;32:459-65.

15. Cervellati S, Bettini N, Moscato M, Gusella A, Dema E, Maresi R. Surgical treatment of spinal deformities in Duchenne muscular dystrophy: a long term follow-up study. Eur Spine J 2004;13:441-8.

16. Eagle M, Bourke J, Bullock R, et al. Managing Duchenne muscular dystrophy: the additive effect of spinal surgery and home nocturnal ventilation in improving survival. Neuromuscul Disord 2007;17:470-5.

17. Kennedy JD, Staples AJ, Brook PD, et al. Effect of spinal surgery on lung function in Duchenne muscular dystrophy. Thorax 1995;50:1173-8.

18. Kinali M, Messina S, Mercuri E, et al. Management of scoliosis in Duchenne muscular dystrophy: a large 10-year retrospective study. Dev Med Child Neurol
2006;48:513-8.

19. Estrup C, Lyager S, Noeraa N, Olsen C. Effect of respiratory muscle training in patients with neuromuscular diseases and in normals. Respiration 1986;50:36-43.

20. Nava S, Ambrosino N, Crotti P, Fracchia C, Rampulla C. Recruitment of some respiratory muscles during three maximal inspiratory manoeuvres. Thorax 1993;48:702-7.

21. Nicot F, Hart N, Forin V, et al. Respiratory muscle testing: a valuable tool for children with neuromuscular disorders. Am J Respir Crit Care Med 2006;174:67-74.

22. Stefanutti D, Benoist MR, Scheinmann P, Chaussain M, Fitting JW. Usefulness of sniff nasal pressure in patients with neuromuscular or skeletal disorders. Am J Respir Crit Care Med 2000;162(4 Pt 1):1507-11.

23. Takaso M, Nakazawa T, Imura T, et al. Surgical management of severe scoliosis with high-risk pulmonary dysfunction in Duchenne muscular dystrophy. Int Orthop 2010;34:401-6.

24. Kim HS, Park JO, Lee HM, et al. Radiographic and functional outcome after surgical management of severe scoliosis in skeletally immature patients with muscular dystrophy. J Spinal Disord Tech 2004; 17:505-10.

25. Kurz LT, Mubarak SJ, Schultz P, Park SM, Leach J. Correlation of scoliosis and pulmonary function in Duchenne muscular dystrophy. J Pediatr Orthop 1983;3:347-53.

26. Oda T, Shimizu N, Yonenobu K, Ono K, Nabeshima T, Kyoh S. Longitudinal study of spinal deformity in Duchenne muscular dystrophy. J Pediatr Orthop 1993;13:478-88.

27. Yamashita T, Kanaya K, Kawaguchi S, Murakami T, Yokogushi K. Prediction of progression of spinal deformity in Duchenne muscular dystrophy: a preliminary report. Spine (Phila Pa 1976) 2001;26:E223-6.

28. Hsu JD. The natural history of spine curvature progression in the nonambulatory Duchenne muscular dystrophy patient. Spine (Phila Pa 1976) 1983;8:7715 . 